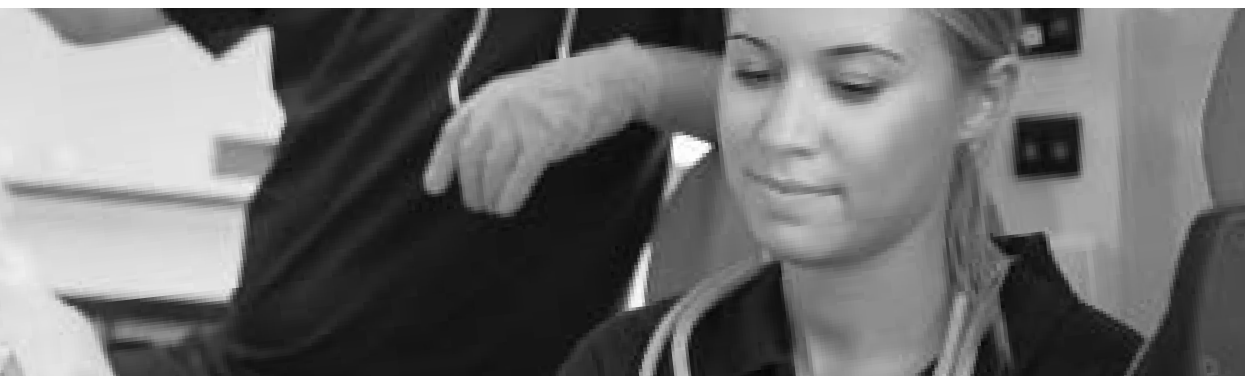

Volume 12 | Issue 5 | Commentary
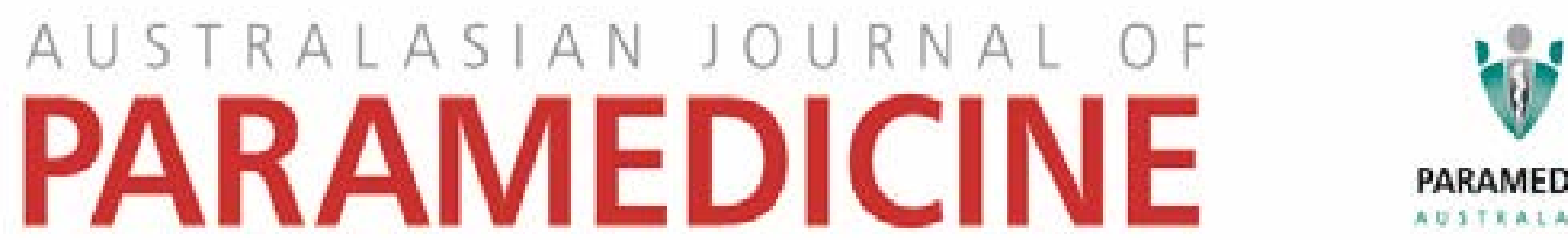

PARAMEDICS

AUSTBALASIA

Pre-hospital medicine: a glimpse of the future

Keron Davison

Queensland Ambulance Service

Malcolm P Forbes

James Cook University 


\title{
Commentary
}

\section{Pre-hospital medicine: a glimpse of the future}

\author{
Keron Davision, BHlthSci(Paramedic) is an Advanced Care Paramedic ${ }^{1}$, Malcolm P Forbes MBBS is a Medical \\ Registrar and Research Associate ${ }^{2,3}$
}

\author{
Affiliation: \\ ${ }^{1}$ Queensland Ambulance Service \\ ${ }^{2}$ Royal Australasian College of Physicians \\ ${ }^{3}$ James Cook University, College of Medicine and Dentistry \\ Corresponding Author: Keron Davison, kerondavison@hotmail.com
}

The availability and utilisation of technology in pre-hospital medicine has increased exponentially in the past half-century and will continue to do so. New technologies, including telepresence, cognitive computing, artificial intelligence (Al), robotics and automation, will revolutionise the way we practise in the 21 st century. In order to maintain relevance and continue to provide exceptional patient care, we must stay abreast of technological advances, critically appraise new technologies, and be early adapters to change.

The scope of practice of paramedics has developed significantly over the past century, with expanding scope of practice allowing timely intervention and enhanced patient care (1). Bachelor degrees are now compulsory entry in many services, and postgraduate study is becoming the norm. Paramedics in Australia have evolved from drivers and bearers into clinicians delivering advanced, life-saving interventions, in time critical and taxing circumstances (2).

Over the coming decades, paramedics are likely to have their core driving and bearing skills replaced by computer-controlled technology. Autonomous vehicle trials are either planned or currently underway in the several countries, including Australia (3-5). If these trials are successful, it is only a matter of time before trials of driverless ambulances will begin.

Driverless ambulances potentially offer advantages over vehicles with a human driver. They could provide the hardware to expand the productivity of existing crew availability. Paramedics would no longer have to spend much of their valuable clinician time behind the wheel. Instead, the majority of crews could act as single responders for lower acuity cases and use this time for patient contact. In the setting of more critical, higher acuity cases, multiple units can be dispatched accordingly. This would expand on the existing models of single response care, and provide much greater area coverage with scarce resources.

When multiple paramedics are required for treatment en route to hospital, the empty ambulances could utilise the 'platooning' technology already in trial with the United States military (6). This technology works by creating a convoy of driverless vehicles following a single lead vehicle. This technique could ensure no time is wasted having to return the supporting officers to their vehicles. By the time this technology reaches civilian ambulance services, it is likely to have been tested in an operational military environment, establishing it within its role.

With the correct planning, it would be possible that unmanned ambulances could return to a central workshop/warehouse facility at the end of each shift. Here they could be refuelled, restocked, aseptically cleaned, and quality control inspected before then returning to their place of duty in anticipation of their next shift. This would reduce the significant opportunity cost currently expended on pre- and post-shift checks by paramedics. Essentially paramedics could step onto the car at the start of a shift, and off again at the end.

In terms of wider impact, it is expected that driverless car technology will lead to a significant reduction in road traffic accidents (7). This in turn will lead to a reduction in traffic related cases requiring an ambulance, if the prediction becomes reality. This will ultimately change the way we do business. 
The intelligent utilisation of cognitive computing, and early generation Al technology such as D-wave quantum computing (8), and IBM's Watson system (9) can be combined with the driverless fleet. This can greatly increase fleet optimisation by control centres. Complex algorithms can be easily utilised to place crews in the best position to provide the most rapid response to incoming cases. This will ultimately lead to an increase in productivity, as there would be less time wasted. This would be a largely passive process on behalf of the paramedic and dispatchers, which would lead to a reduced cognitive load on officers.

The advent of robotic lift assistance devices will render the bearer part of the job largely obsolete. The remit of paramedics will evolve into a purely clinical role. Ship builders in South Korea are already using exoskeleton technology in order to lift otherwise dangerously large loads (10). Re-walk exoskeleton is currently being trialled in order to assist paraplegics to walk upright (11). These technologies will only need to be adapted and not reinvented to relieve paramedics of the physical burden of heavy lifts they currently experience. This is likely to lead to a reduction in injury rates, such as muscular stress from lifting and carrying (12).

These changes are likely to meet opposition from some paramedics who consider these skills a core part of their professional identity, however they will need to concede, for the good of the public and professional interest.

Telemedicine in pre-hospital medicine is already in use, albeit in its infancy (13). When applied fully, it has the potential to dramatically change the interventions that can be performed in the pre-hospital setting. It will allow collaboration between the treating emergency physician and treating paramedic to deliver time-critical interventions prior to arrival in the emergency room, improving patient outcomes (14).

Queensland Ambulance Service is currently in the process of issuing tablets to all officers. These tablets typically come with a $4 \mathrm{G}$ connection. This will give the crews the ability to obtain a live video feed where the connection allows. This has the potential to allow a face-to-face consult with a physician to occur, allowing early assessment and intervention. This has the potential to vastly increase the scope of pre-hospital care provided.

This also presents a real opportunity for 'at home' physician authorised treatment and discharge without the need for transport to a receiving facility. With correct implementation and training, paramedics can have the majority of the assessment and treatment in place prior to consultation. This could lead to a significant improvement in medical productivity.

It would not only be mobile telepresence technology that would prove its value. Ambulance services across Australia have existing valuable real estate in many areas, particularly remote. Casualty rooms can be upgraded to allow a portal to medical treatment in underserviced areas. The paramedics at these stations would have the opportunity to significantly expand their scope, whilst practising under the guidance of a supervising physician. Expert communication, including the use of advanced collaboration strategies, is needed for this technology to be utilised effectively (15).

Telemedicine is already in use in the pre-hospital setting, and while the majority of studies demonstrate some positive impact on emergency medical care, there are few definitive studies that have demonstrated whether it has an effect on clinical outcomes (16).

Technologies currently being trialled will allow the telepresence experience to become more immersive. Wearable technology such pivothead glasses (17) will allow consulting physicians to have "point of view" imaging from the paramedic's perspective. Virtual reality headsets such as the Oculus Rift (18), can combine with on scene telepresence robots to give the full telepresence experience to those in ED giving 360 degree, 3D vision to the wearer, along with a degree of control, to navigate the environment (19). Blue tooth stethoscopes (20) can allow doctors to remotely auscultate. Sensory feedback systems similar to those employed on the Da Vinci surgical machine will allow doctors to palpate patients remotely (21).

Australia is the ideal location for the roll out of these new tools. The remoteness of many Australian communities, which have a paramedic present, combined with the advanced medical facilities and technical support in metropolitan areas will allow integrated patient care networks to evolve, creating greater equity between city and rural areas with regards to access to healthcare (22).

With leadership in this arena, Australia could be an early adopter of technology that will facilitate improved health outcomes, greater access to top quality care in remote areas and greater collaboration between emergency medical personnel. The implementation of this will need to be very careful and methodical due to the novelty of the field. This research and development should ideally be undertaken by the people most familiar with the environment. 
The use of the scientific method using sound methodologies combined with solid business support models will be essential. It will also be vital to use qualitative methods to ensure patient and practitioner satisfaction. It would be counterproductive to utilise systems which nobody wants.

We are now in the position to design futuristic health networks, which can divert resources from bricks and mortar infrastructure to human resources. Given the level of investment necessary, we must progress cautiously in this new and exciting area. But progress we must.

\section{References}

1. B Williams TB, A Onsman. From stretcher-bearer to paramedic: The Australian paramedics' move toward professionalisation. Australasian Journal of Paramedicine. 2012;7(4).

2. Hou XY, Rego J, Service M. Review article: Paramedic education opportunities and challenges in Australia. Emerg Med Australas 2013;25(2):114-9.

3. Catapult Transport Systems. LUTZ Pathfinder Automated Pods 2015. Available at: https://ts.catapult.org.uk/pods [Accessed 19 August 2015].

4. Research AT. ARRB announces first driverless car trials on Australian roads 2015. Available at: www.arrb.com.au/Home/ News.aspx?newsID=227 [Accessed19 August 2015].

5. Google. Google Self Driving Car Project 2015. Available at: www.google.com/selfdrivingcar/where/ [Accessed 19 August 2015].

6. Martin L. Autonomous Mobility Appliqué System (AMAS) 2015. Available at: www.lockheedmartin.com/us/products/amas1. html [Accessed 19 August 2015].

7. Litman T. Autonomous Vehicle Implementation Predictions. Victoria Transport Policy Institute. 2014;28.

8. D-wave Quantum Computing Company. Applications - Optimization 2015 Available at: www.dwavesys.com/quantumcomputing/applications [Accessed 19 August 2015].

9. IBM. What is Watson? 2015 Available at: www.ibm.com/smarterplanet/us/en/ibmwatson/what-is-watson.html [Accessed 19 August 2015].

10. DSME. New Technology List. Available at: www.dsme.co.kr/epub/technology/technology0108.do?index=7 [Accessed 19 August 2015].

11. ReWalk. New ReWalk ${ }^{\text {TM }}$ Personal 6.0 2015. Available at: http://rewalk.com/rewalk-personal-3/ [Accessed 19 August 2015].

12. Maguire BJ, O'Meara PF, Brightwell RF, O'Neill BJ, Fitzgerald GJ. Occupational injury risk among Australian paramedics: an analysis of national data. Med J Aust 2014;200(8):477-80.

13. Nangalia V, Prytherch DR, Smith GB. Health technology assessment review: remote monitoring of vital signs--current status and future challenges. Crit Care 2010;14(5):233.

14. Rörtgen D, Bergrath $S$, Rossaint $R$, et al. Comparison of physician staffed emergency teams with paramedic teams assisted by telemedicine-a randomized, controlled simulation study. Resuscitation 2013;84(1):85-92.

15. Amadi-Obi A, Gilligan P, Owens N, O'Donnell C. Telemedicine in pre-hospital care: a review of telemedicine applications in the pre-hospital environment. Int J Emerg Med 2014;7(1):1-11.

16. Sonnenwald DH, Söderholm HM, Welch GF, Cairns BA, Manning JE, Fuchs H. Illuminating collaboration in emergency health care situations: paramedic-physician collaboration and 3D telepresence technology. Information Research 2014;19(2). Available at: www.informationr.net/ir/19-2/paper618.html\#.VeBPdNOqqkp

17. pivothead. 2015 [cited 2015 20/08]. Available at: www.pivothead.com/

18. Oculus. Rift 2015. Available at: www.oculus.com/rift [Accessed 19 August 2015].

19. Robotics R. What is KUBI 2015. Available at: www.revolverobotics.com/ [Accessed 20 August 2015],

20. Littman. Telemedicine 2015 Available at: www.littmann.com/wps/portal/3M/en_US/3M-Littmann/stethoscope/telemedicine/

21. Schneider C, Baghani A, Rohling R, Salcudean S. Remote ultrasound palpation for robotic interventions using absolute elastography. Medical image computing and computer-assisted intervention: MICCAI International Conference on Medical Image Computing and Computer-Assisted Intervention. 2012;15(Pt 1):42-9.

22. O'Meara PF, Tourle V, Stirling C, Walker J, Pedler D. Extending the paramedic role in rural Australia: a story of flexibility and innovation. Rural Remote Health 2012;12(2):1978.

Disclaimer: The views expressed in this commentary are those of the authors and do not necessarily reflect the policies of the institutions where they are employed. 